\title{
Producción de agua potable a partir de agua de mar por destilación y ósmosis inversa
}

Mario Rojas Delgado

En el presente artículo se examinan las tecnologías de mayor aplicación en la actualidad para producir agua fresca a partir de agua de mar, considerando aspectos técnicos y costos de producción. Se comparan los sistemas de producción de agua potable por tratamiento convencional, destilación y ósmosis inversa, basado en información que permita seleccionar el proceso adecuado para un proyecto industrial y, en general, para uso público. 


\section{Introducción}

Debe considerarse que tanto en el Perú como en otros países hay zonas que requieren utilizar fuentes alternativas de agua, no obstante que el costo unitario es todavía superior en comparación con el de las fuentes usuales. Históricamente ha sido un reto permanente para la ingeniería peruana desarrollar suficientes fuentes de agua para la población, que se distribuye a lo largo de aproximadamente 2.800 kilómetros de litoral, y que vive frente a un océano pero carece del servicio de agua potable para la alimentación, salud, agricultura y para el desarrollo industrial local. El propósito principal es dar a conocer cuáles son las tecnologías más utilizadas actualmente en el Perú y en otros países, y proporcionar datos técnicos y de costos que permitan comparar y seleccionar los sistemas viables que puedan aplicarse en nuestro país.

Se presentan diagramas de bloque y diagramas de flujo de uso industrial y comercial para la producción de agua por desalinización, para que el proyectista académico o comercial cuente con una rápida referencia en la preparación de un perfil de proyecto o estudio tecnológico que puede ser complementado con las referencias bibliográficas.

\section{Generalidades}

Se estima que existen en el mundo unas 7.500 plantas de desalinización de agua salada, localizándose un $60 \%$ en el Medio Oriente. La planta más grande en Arabia Saudita produce $480 \mathrm{mil} \mathrm{m} 3$ día de agua desalada. En el continente americano está el $12 \%$ de la capacidad mundial, ubicada principalmente en Florida, Estados Unidos, y en el Caribe.

\section{Tecnologías para la desalinización de agua}

La desalinización es un proceso que extrae los minerales disueltos en el agua, los cuales expresados en sus elementos constituyentes se indican en la tabla $\mathrm{N}^{0} 1$. Las tecnologías más utilizadas han sido ósmosis inversa (OR), destilación (D), electrodiálisis (ED), y congelamiento al vacío (CV). Sólo las dos primeras han sido consideradas por empresas, municipios y estados como las más aprovechables. Adicionalmente, se han desarrollado los procesos de cogeneración (PC). 
La ósmosis inversa consiste en bombear agua a través de membranas permeables, separando las sales y el agua, tal como se muestra en la figura $\mathrm{N}^{0} 1$. El agua cruda es pretratada para remover partículas que pueden bloquear los poros de las membranas.

La destilación consiste en calentar el agua cruda y evaporar el agua pura separando los minerales disueltos. Los métodos comunes empleados son: sistema flash multiefecto o con reciclo (FME), sistema multiefecto (ME), y sistema por compresión de vapor (CV). Estos procesos se muestran en las figuras $\mathrm{N}^{\mathrm{o}} 2,3 \mathrm{y}$ 4, respectivamente. En el sistema FME el agua cruda es calentada y luego se baja la presión para obtener el "flash" (vaporización instantánea). En el sistema ME el agua cruda pasa por un sistema de evaporadores instalados en serie. El sistema VC comprende la evaporación a partir del agua cruda y comprime el vapor para calentarlo y usarlo como fuente de energía para evaporar agua adicional.

Los procesos de cogeneración comprenden la producción de energía mediante una turbina accionada por vapor o gas proveniente del sistema de evaporación en la producción de agua pura a partir del agua de mar. En las figuras $\mathrm{N}^{\mathrm{o}} 11,12$ y 13 se muestran los detalles de estos procesos.

\section{Uso de energía y costo}

Como referencia rápida se estima que el rango de uso de energía para obtener agua fresca a partir de agua salada es entre 3-6 kW/hora con tecnologías avanzadas con respecto al estándar histórico.

En cuanto al costo de agua fresca obtenida por los procesos de desalinización se estima como mínimo $\$ 3 / \mathrm{m}^{3}$.

\section{Tecnología convencional}

El término tecnología convencional se refiere a las operaciones y procesos para la producción de agua potable a partir de fuentes de agua superficiales (ríos) y fuentes de agua subterráneas en aquellas zonas o regiones susceptibles de ser explotadas por su topografía y orografía, que permitan operaciones a gran escala para obtener un costo de agua fresca relativamente bajo. En el Perú existen a nivel regional este tipo de empresas con plantas de producción de agua potable, como es el caso de la 
planta La Atarjea, perteneciente a Sedapal, que puede ofrecer tarifas de agua para uso residencial a partir aproximadamente de $\$ 0,30 / \mathrm{m}^{3}$.

\section{Tecnología de ósmosis inversa}

Los procesos de ósmosis inversa se basan en el empleo de sistemas de membranas permeables para separar el agua de sales disueltas en el agua salada. Se aplican tanto a agua de mar como a la categoría de agua salobre. Como referencia se presentan dos esquemas tecnológicos, uno para servicios de menor escala (uso residencial o establecimientos comerciales menores) y el otro para una mayor escala de producción de agua fresca:

- Operación de ósmosis inversa

- Planta de ósmosis inversa

Los diagramas de flujo para dichos procesos se presentan en las figuras $\mathrm{N}^{0} 5$ y 6 , respectivamente.

\section{Destilación de agua de mar}

Los procesos en general de destilación de agua salada para producir agua fresca se basan en la evaporación de agua con sistemas de muy variado diseño, que tienen como fin primordial reducir los costos de operación. Como referencia se presentan las siguientes tecnologías de actualidad que están empleándose:

- Sistema flash multietapa

- Sistema flash de múltiples etapas con una pasada

- Evaporador por reciclo de calor

- Desalinización por múltiple efecto con termo compresión

En todos estos casos el destilado condensado constituye el producto denominado agua fresca, que todavía es susceptible de tener un postratamiento. En las figuras $N^{\circ}$ 7, 8, 9 y 10 se presentan los diagramas de flujo de dichos procesos respectivamente. La fuente primaria de energía es combustible fósil no renovable.

\section{Destilación de agua de mar con energía solar}

En estos procesos se consideran los mismos tipos de equipamiento y diseño que tienen los sistemas de destilación, con la diferencia que se utiliza energía solar clasificada como energía 
renovable hasta cierto punto. Este tipo de procesos dependerá de la disponibilidad de horas con radiación solar en la región en la cual se planea instalar la planta. Es factible encontrar estos procesos en embarcaciones marítimas.

\section{Tecnología de cogeneración}

Estos procesos producen agua fresca a partir de agua salada, y simultáneamente producen energía eléctrica empleando el vapor de agua como sustancia de trabajo que acciona una turbina, la cual a su vez acciona un generador de corriente eléctrica. El vapor condensado después de accionar la turbina es el agua que, previo tratamiento final, puede emplearse como agua potable. Se integra este sistema híbrido con el empleo de unidades de ósmosis inversa. Se presentan los siguientes procesos de referencia:

- Cogeneración de ciclo combinado y ósmosis inversa

- Cogeneración de turbina de vapor y ósmosis inversa

- Cogeneración de turbina de gas y ósmosis inversa

Se presentan los diagramas de flujo para estos procesos en las figuras $\mathrm{N}^{\mathrm{o}} 11,12$ y 13 , respectivamente.

\section{Comparación de tecnologías}

Se considera que las tecnologías predominantes son las de ósmosis inversa y la de destilación, algunos proyectistas consideran para el análisis el consumo de energía de los sistemas comerciales para producir agua fresca a partir de agua salada. En la tabla $\mathrm{N}^{\mathrm{o}} 2$ se presentan algunas cifras de consumo de energía ( $\mathrm{kW} / \mathrm{m}^{3}$ agua fresca) para plantas de instalación reciente.

Otro factor importante para el análisis es el costo de producción de agua fresca ( $\$ / \mathrm{m}^{3}$ agua fresca); tomando como referencia el plan de nuevas o proyectadas plantas para desalinizar agua salada del estado de California, se muestran algunos datos de costos en la tabla $\mathrm{N}^{\circ}$ 3. Esta referencia es muy útil por la cantidad de proyectos planeados para la década del noventa en esa región con tecnología actual.

\section{Opciones de aplicación en zonas costeras del Perú}

Hay empresas industriales ubicadas tanto en el centro como en el sur del país que cuentan con plantas de tamaño comercial 
para la producción de agua fresca por desalinización de agua de mar. Se puede citar a una empresa minera del sur que opera con una planta desde hace 46 años y en la actualidad produce $2.800 \mathrm{~m}^{3} /$ día. En zonas urbanas se puede contar con agua potable a $\$ 0,30 / \mathrm{m}^{3}$ con sistemas convencionales en comparación con el costo de $\$ 3,0 / \mathrm{m}^{3}$ por los sistemas de desalinización de agua de mar, lo que constituye una barrera para tales tecnologías; sin embargo, el estudio económico-social debe contemplar las consecuencias y costos por la carencia de fuentes de agua que afectan directamente a la población, especialmente en la región costera, debido a las altas inversiones que se requieren para los proyectos de irrigación para usar los sistemas convencionales.

\section{Impacto ambiental y energético}

El proyectista deberá considerar en el proyecto aspectos relativos al impacto ambiental al extraer grandes cantidades de sales del medio marino, y, así mismo, el impacto de consumir energía de fuentes no renovables como es el petróleo. Alternativamente debería contemplarse el uso de carbón en zonas de yacimientos cercanos al litoral, como por ejemplo el valle del Alto Chicama.

\section{Conclusiones}

- Las tecnologías de mayor uso en la actualidad para producir agua potable a partir de agua de mar son la ósmosis inversa y la destilación.

- En el mundo, el costo de producción de agua potable por desalinización es aceptable, sin embargo en el Perú dicho costo es todavía demasiado alto para nuestra realidad.

- Se debe investigar y desarrollar el empleo del carbón como alternativa para reducir el costo de la desalinización del agua de mar. 


\section{Bibliografía}

Boyle Engineering Corp. for California Urban Water Agencies of Desalination for Urban Water Supply 1991. $<$ http:/ceres.ca.gov/coastalcom/desalrpt/dsynop.html> Khan, A.H.

Desalination Processes and Multi Stage Flash Distillation Practice. New York: Ed. Elsiever, 1986.

Rochem Separation Systems. RO-Water Desalination, 1991. $<$ http:/ceres.ca.gov/coastalcom/desalrpt/dchapt1.html>

Muslin, D. U.S. Navy, Environmental Division, 1993. $<$ http:/ceres.ca.gov/coastalcom/desalrpt/dbiblio.html>

Wilson, T. Pacific Gas \& Electric Company. Diablo Canyon Power Plant, 1993.

$<$ http:/ceres.ca.gov/coastalcom/desalrpt/dbiblio.html>

Top Ten Desalination Web Sites, Updated May 5, 1998:

- Aqua - Chem World Information

- European Desalination Center Society

- International Desalination Association

- Middle East International Business Magazine

- Middle East Desalination Research Center

- Seawater Desalination in California

- U.S. Bureau Reclamation

- U.S. Water News

- Water Online Times

- World Wide Water

$<$ http://www2.hawaii.edu/ nabil/file-eds.htm> 
Tabla No 1

Principales elementos del agua de mar

\begin{tabular}{|c|c|c|c|}
\hline Elemento & $\begin{array}{l}\text { Concentración } \\
\text { (mg/l) }\end{array}$ & Elemento & $\begin{array}{l}\text { Concentración } \\
\text { (mg/l) }\end{array}$ \\
\hline Oxygen & $8,57 \times 10+5$ & Iron & $1 \times 10-2$ \\
\hline Hydrogen & $1,08 \times 10+5$ & Indium & $<2 \times 10-2$ \\
\hline Chlorine & $1,90 \times 10+4$ & Molybdenum & $1 \times 10-2$ \\
\hline Sodium & $1,05 \times 10+4$ & Zinc & $1 \times 10-2$ \\
\hline Magnesium & $1,35 \times 10+3$ & Nickel & $5,4 \times 10-3$ \\
\hline Sulfur & $8,85 \times 10+2$ & Arsenic & $3 \times 10-3$ \\
\hline Calcium & $4,00 \times 10+2$ & Copper & $3 \times 10-3$ \\
\hline Potassium & $3,80 \times 10+2$ & Tin & $3 \times 10-3$ \\
\hline Bromine & $6,5 \times 10+1$ & Uranium & $3 \times 10-3$ \\
\hline Carbon & $2,8 \times 10+1$ & Krypton & $2,5 \times 10-3$ \\
\hline Strontium & $8.1 \times 10+0$ & Manganese & $2 \times 10-3$ \\
\hline Boron & $4,6 \times 10+0$ & Vanadium & $2 \times 10-3$ \\
\hline Silicon & $3 \times 10+0$ & Titanium & $1 \times 10=3$ \\
\hline Fluorine & $13 \times 10+0$ & Cesium & $5 \times 10-4$ \\
\hline Argon & $6 \times 10-1$ & Cerium & $4 \times 10-4$ \\
\hline Nitrogen & $5 \times 10-1$ & Antimony & $3,3 \times 10-4$ \\
\hline Lithium & $1,8 \times 10-1$ & Silver & $3 \times 10-4$ \\
\hline Rubidium & $1,2 \times 10-1$ & Yttium & $3 \times 10-4$ \\
\hline Phosphorus & $7 \times 10-2$ & Cobalt & $2,7 \times 10-4$ \\
\hline lodine & $6 \times 10-2$ & Neon & $1,4 \times 10-4$ \\
\hline Barium & $3 \times 10-2$ & Cadmium & $1,1 \times 10-4$ \\
\hline Aluminum & $1 \times 10-2$ & Tungsten & $1 \times 10-4$ \\
\hline
\end{tabular}

Fuente: Handbook of Chemistry and Physics, 64th Edition. CRC Press, 1984. 
Tabla No 2

Uso de energía

\begin{tabular}{ll}
\hline Flash multietapa (FME) & $2,8-5,6 \mathrm{kWh} / \mathrm{m} 3$ \\
Destilación multiefecto (DME) & $2,0-4,0 \mathrm{kWh} / \mathrm{m} 3$ \\
Compresión de vapor (CV) & $8,1-12,1 \mathrm{kWh} / \mathrm{m} 3$ \\
Ósmosis inversa (OR) - Monopaso & $4,7-8,9 \mathrm{kWh} / \mathrm{m} 3$ \\
Ósmosis inversa (OR) - Doble paso & $5,2-9,7 \mathrm{kWh} / \mathrm{m} 3$ \\
\hline
\end{tabular}

Fuente: Wilf, M. Hydronautics, 1991. <http:/ceres.ca.gov/coastalcom/desalrpt/dbiblio.html>

Tabla $\mathbf{N}^{\circ} 3$

Costo de agua para plantas de desalinización

\begin{tabular}{lc}
\hline Plantas de desalinización de agua de mar & Costo $\left(\$ / \mathbf{~ m}^{3}\right)$ \\
\hline$-\quad$ Chevron Gaviota Oil and Gas Processing Plant & 3,23 \\
- City of Morro Bay & 1,41 \\
- City of Santa Barbara & 1,53 \\
- Marin Municipal District & $1,29-1,37$ \\
- Metropolitan Water District (MWD) of Southern California & 0,57 \\
- Monterey Bay Aquarium & 1,45 \\
- PG \& E Diablo Canyon Power Plant & 1,62 \\
- San Diego County Water Authority (South Bay Desalination Plant) & $0,89-1,05$ \\
- SCE, Santa Catalina Island & 1,62 \\
- U.S. Navy, San Nicolas Island & 4,85 \\
Otras fuentes de agua & \\
- Ciudad de Santa Bárbara & \\
$\quad$ Lago Cachuma & 0,03 \\
$\quad$ Agua subterránea & 0,16 \\
$\quad$ Agua subterránea de montañas & $0,48-0,57$ \\
$\quad$ Ampliación de reservorio & 0,77 \\
$\quad$ Enlace con proyecto estatal de agua & 1,05 \\
$\quad$ Proyecto estatal temporal de agua repartida via MWD & 1,86 \\
- Metropolitan Water District (MWD) of Southern California & 0,57 \\
$\quad$ Río Colorado & 0,02 \\
$\quad$ Proyecto de agua de California & 0,16 \\
$\quad$ Imperial Irrigation District & 0,11 \\
Proyecto de almacenamiento de agua & 0,07 \\
- San Diego County & \\
$\quad$ MWD & 0,22 \\
$\quad$ Nuevos proyectos de agua & $0,48-0,57$ \\
\hline
\end{tabular}

Fuente: Muir, B. Metropolitan Water District of Southern California, Public information, 1991.

Hess, G.; R. Yamada. San Diego County Water Authority, 1991.

<http:/ceres.ca.gov/coastalcom/desalrpt/dbiblio.html> 


\section{Figura № 1}

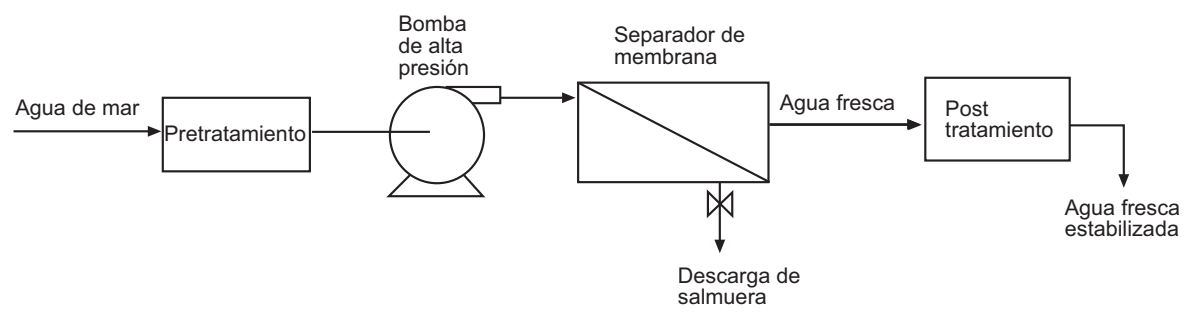

Figura $\mathrm{N}^{\circ} 2$

Proceso multietapa con reciclo

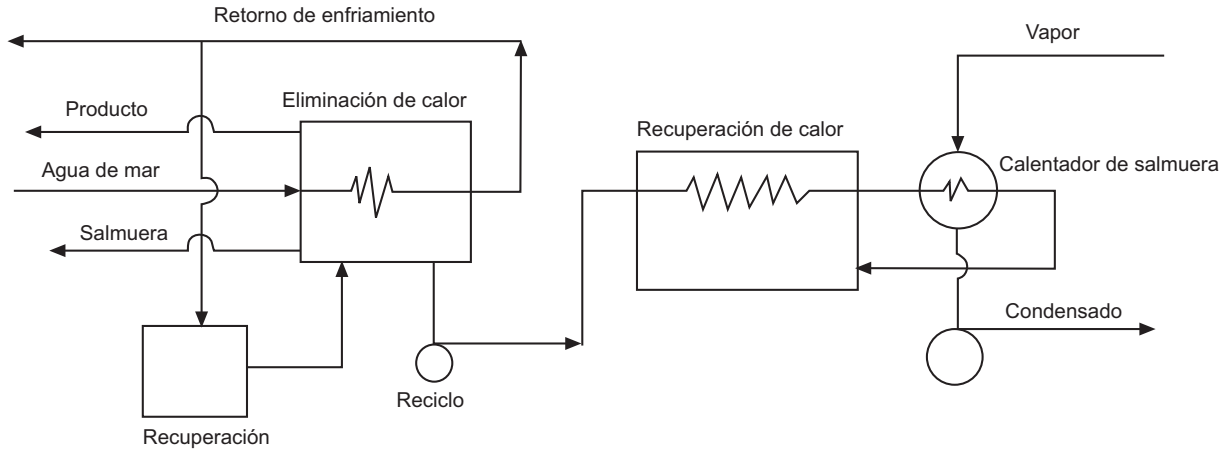


Figura $N^{\circ} 3$

Múltiple efecto

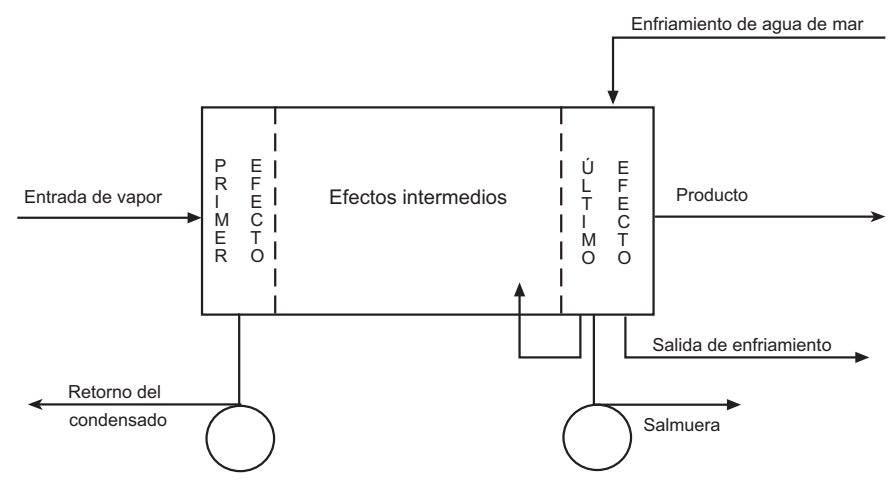

Figura $\mathrm{N}^{\circ} 4$

Compresión de vapor

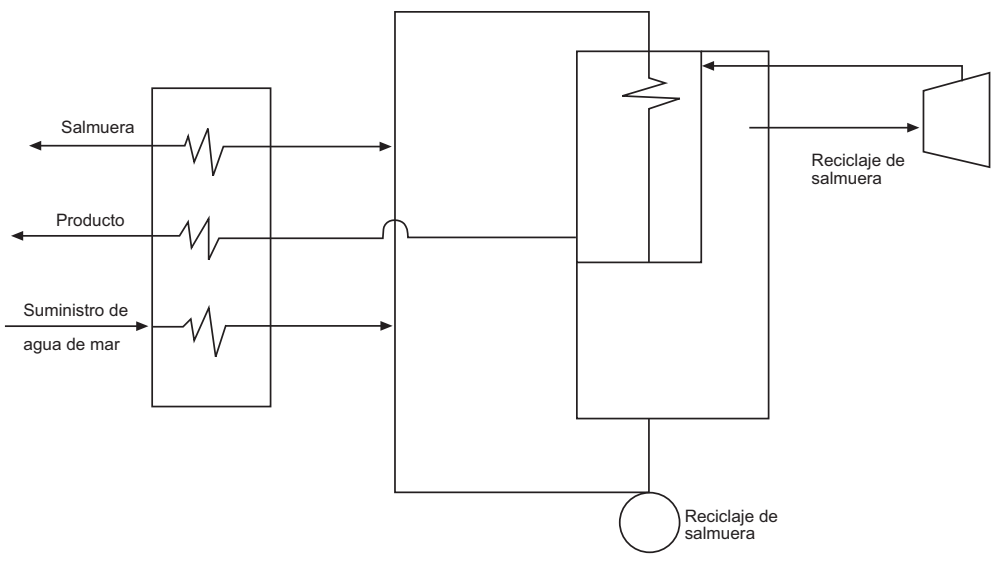


Figura $\mathrm{N}^{\circ} 5$

Operación de ósmosis inversa

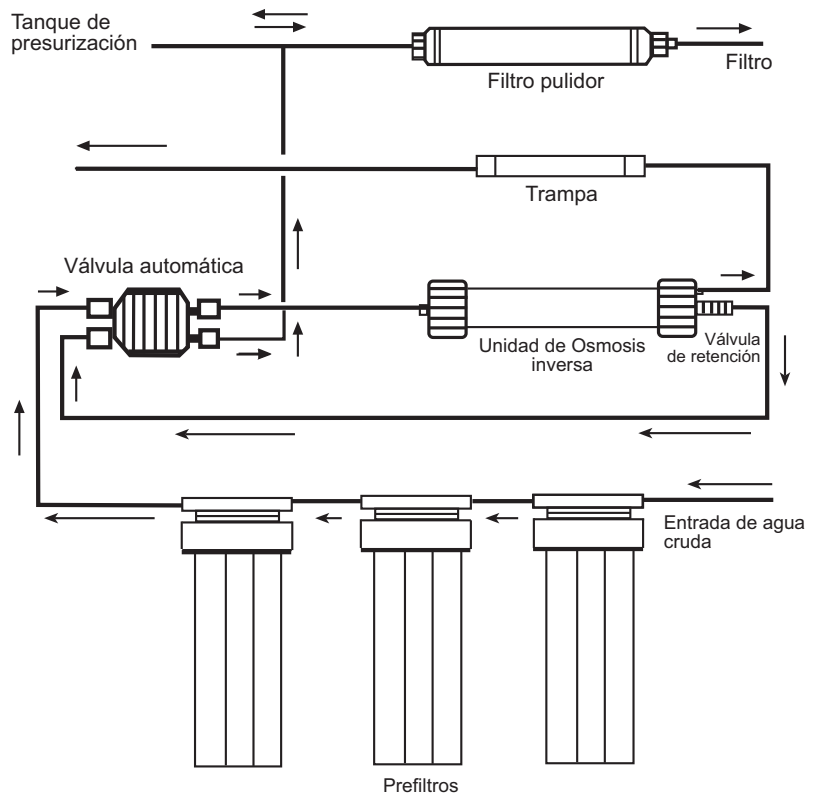

Figura $N^{\circ} 6$

Planta de ósmosis inversa

Agua cruda

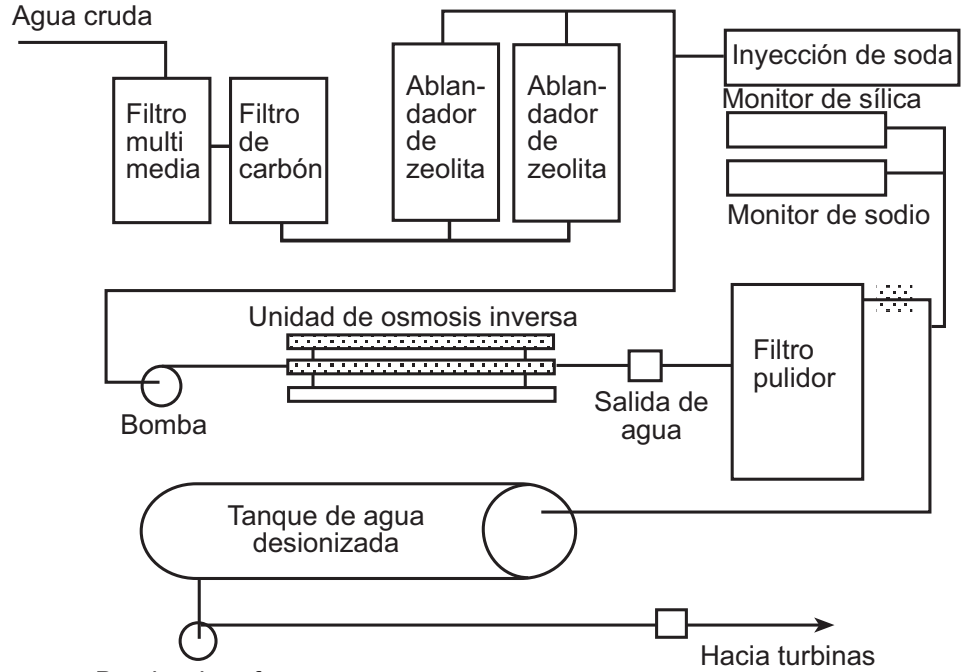

Bomba de refuerzo 
Figura $\mathrm{N}^{\circ} 7$

Sistema flash multietapa

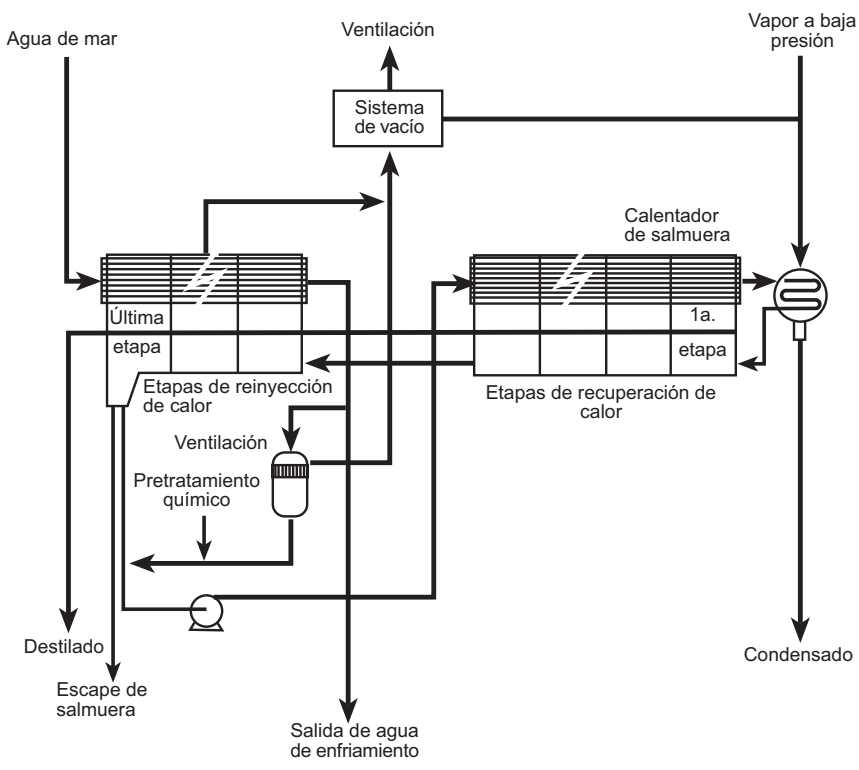

Figura $\mathrm{N}^{\circ} 8$

Sistema de flasheo de múltiples etapas con una pasada

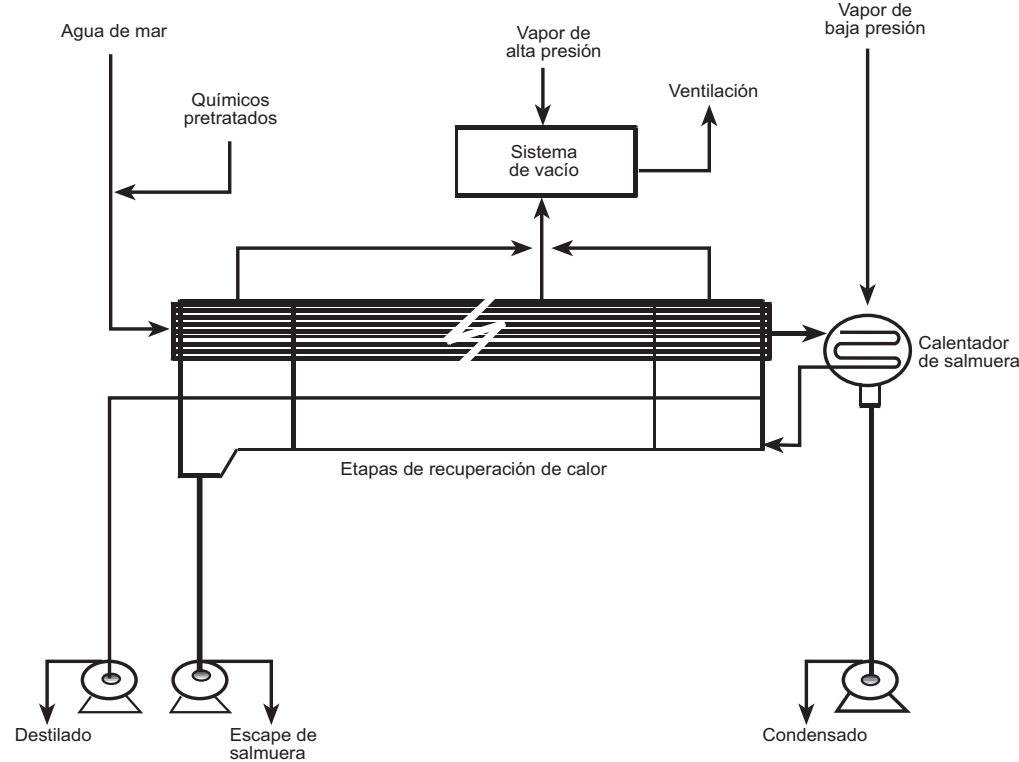




\section{Evaporador por reciclo de calor}

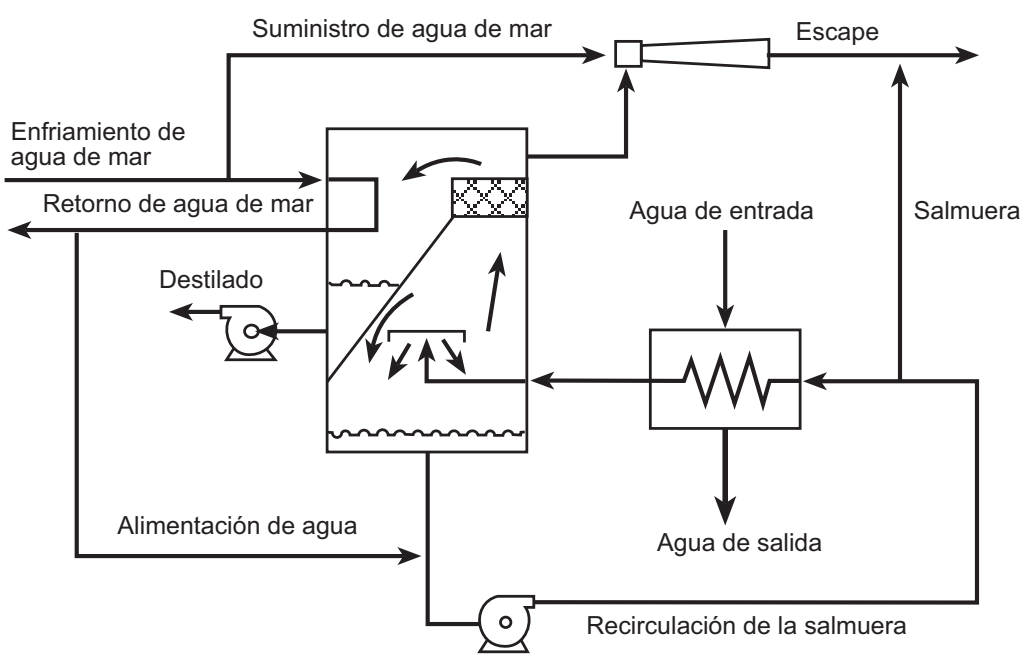

Figura No 10

Desalinización por múltiple efecto con termocompresión

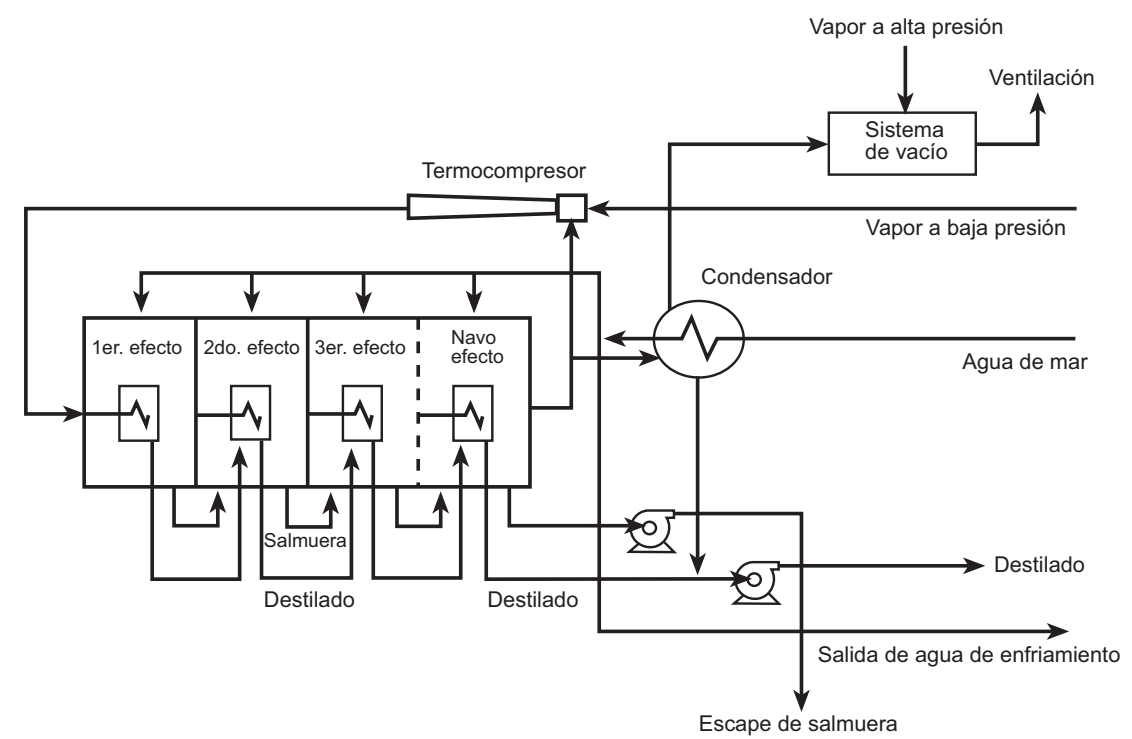


Figura $N^{\circ} 11$

Cogeneración de ciclo combinado y

ósmosis inversa

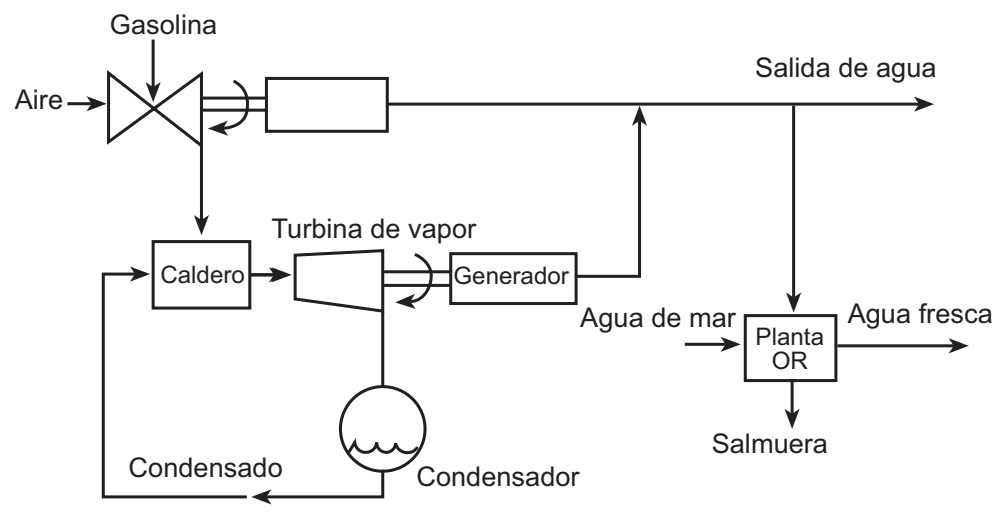

Figura $\mathbf{N}^{\circ} 12$

Cogeneración de turbina de vapor y ósmosis inversa

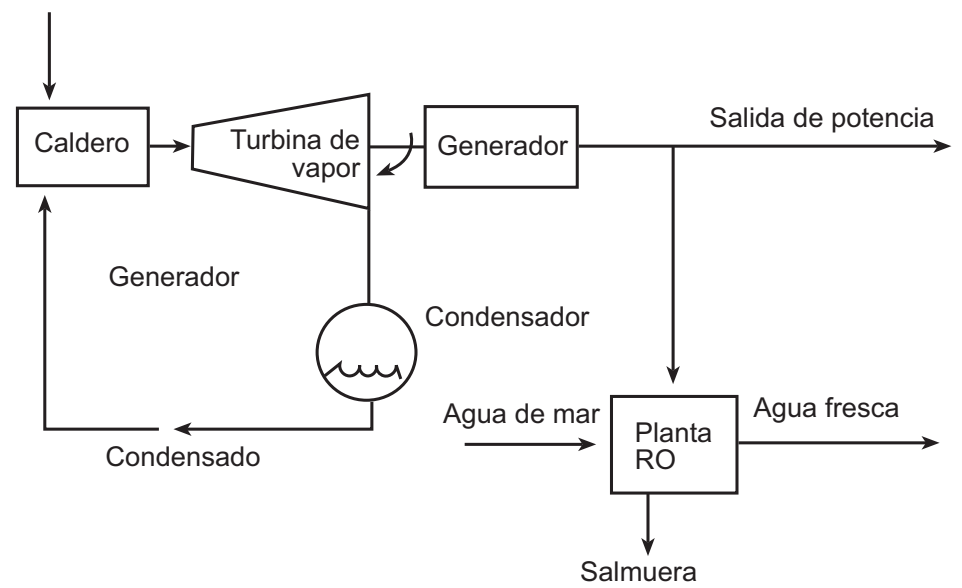


Figura $\mathbf{N}^{\circ} 13$

Cogeneración de turbina de gas y ósmosis inversa

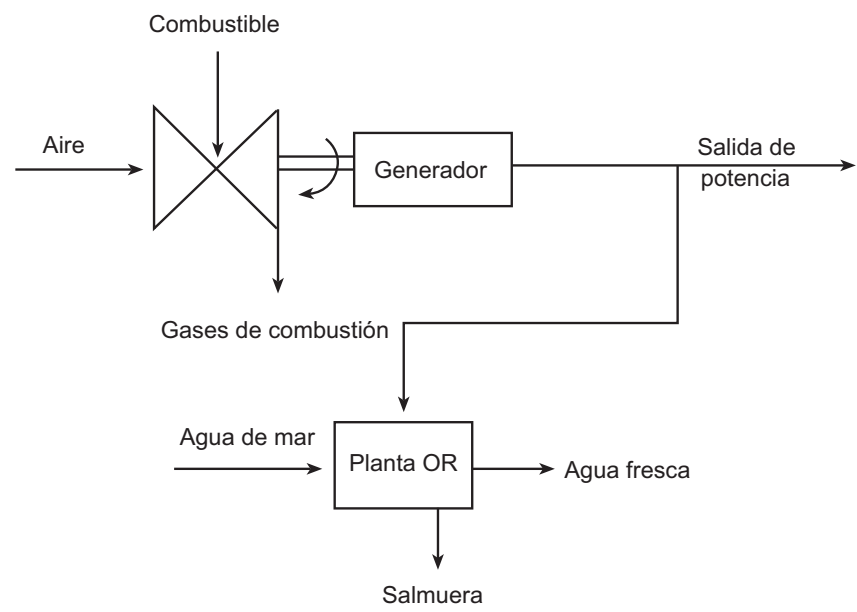

